\title{
BLOW-UP BEHAVIOR FOR A SEMILINEAR HEAT EQUATION WITH A NONLINEAR BOUNDARY CONDITION
}

\author{
Sheng-Chen Fu, Jong-Shend Guo And Je-Chiang Tsai
}

(Received December 28, 2001, revised September 9, 2002)

\begin{abstract}
We study the blow-up behaviors of solutions of a semilinear heat equation with a nonlinear boundary condition. Under certain conditions, we prove that the blow-up point occurs only at the boundary. Then, by applying the well-known method of Giga-Kohn, we derive the time asymptotic of solutions near the blow-up time. Finally, we prove that the blow-up is complete.
\end{abstract}

1. Introduction. In this paper, we study the following initial boundary value problem

$$
\begin{aligned}
& u_{t}=u_{x x}+u^{p}, \quad x \in(0,1), \quad t>0, \\
& u_{x}(0, t)=0, \quad u_{x}(1, t)=u^{q}(1, t), \quad t>0, \\
& u(x, 0)=u_{0}(x), \quad x \in[0,1],
\end{aligned}
$$

where $p, q$ are positive constants, and $u_{0}(x)$ is a positive smooth function. For convenience, we always assume that

$$
u_{0}^{\prime}(0)=0, \quad u_{0}^{\prime}(1)=u_{0}^{q}(1) .
$$

We say that the solution $u$ of the problem (1.1) - (1.3) blows up if there is a finite time $T$ such that $\max _{0 \leq x \leq 1} u(x, t) \rightarrow \infty$ as $t \uparrow T$. It has been shown in [11] that the solution $u$ of the problem (1.1) - (1.3) blows up if and only if $\max \{p, q\}>1$. In [11], they also studied the blow-up set and derived the upper and lower bounds for blow-up rate under certain conditions.

A point $x_{0}$ is said to be a blow-up point for $u$ if there is a sequence $\left\{\left(x_{n}, t_{n}\right)\right\}$ such that $x_{n} \rightarrow x_{0}, t_{n} \rightarrow T$, and $u\left(x_{n}, t_{n}\right) \rightarrow \infty$ as $n \rightarrow \infty$. Under certain conditions, it is shown in [11] that the boundary point $x=1$ is the only blow-up point. This phenomenon of blow-up on the boundary has been observed and studied by many authors. We refer the readers to two nice survey papers [4] and [2] and the references cited therein. See also the references cited in [11].

We are concerned with the blow-up behaviors of solutions of the problem (1.1)-(1.3). Hence throughout this work we always assume that $\max \{p, q\}>1$. In the sequel, we shall assume that the solution $u$ of the problem (1.1)-(1.3) blows up at $T<\infty$. First, we study the blow-up set. We prove that blow-up point occurs only at the boundary point $x=1$, if $u_{0}^{\prime} \geq 0$ in $[0,1]$. This improves the results of [11], where the monotonicity of $u$ in time is

2000 Mathematics Subject Classification. Primary 35K60; Secondary 34A12, 35B40, 35K20.

This work was partly supported by the National Science Council of the Republic of China under the contract NSC 89-2115-M-003-014. 
assumed. Furthermore, by deriving some a priori estimates, with the help of the lower and upper bounds for blow-up rate, we can apply the well-known Giga-Kohn transformation (cf. [8]) to derive the time asymptotic of solutions. This gives a more precise information of the blow-up behaviors. We remark here that a similar problem for the case when the heat operator is replaced by the porous medium operator in the half real line has been studied by de Pablo, Quirós and Rossi [3].

The next question is the possibility of continuation of solutions beyond the blow-up time. For more references on this subject, we refer the readers to the paper [5] and some references listed there. We show that the blow-up for the problem (1.1) - (1.3) is complete, i.e., solutions blowing up in finite time will be infinite identically after the blow-up time.

This paper is organized as follows. We study the blow-up set in Section 2. Some a priori estimates are given in Section 3. In Section 4, we study the self-similar solution for the critical case by an ordinary differential equation approach. Then we study the time asymptotic of the solution in Section 5. Finally, in Section 6 we prove that the blow-up is complete.

We thank the referee for helpful comments which improve some results in Section 2.

2. Blow-up set. Let $u$ be the solution of the problem

$$
\begin{aligned}
& u_{t}=u_{x x}+u^{p}, \quad x \in(0,1), \quad 0<t<T, \\
& u_{x}(0, t)=0, \quad u_{x}(1, t)=u^{q}(1, t), \quad 0<t<T, \\
& u(x, 0)=u_{0}(x), \quad x \in[0,1],
\end{aligned}
$$

where $T$ is the blow-up time of $u$. Here as usual we always assume that $u_{0}^{\prime}(0)=0$ and $u_{0}^{\prime}(1)=u_{0}^{q}(1)$. We shall assume that $u_{0}^{\prime} \geq 0$ in $[0,1]$, so that by the maximum principle we have $u_{x}>0$ in $(0,1] \times(0, T)$. We shall modify the method of Friedman and McLeod ([7]) to study the blow-up set.

THEOREM 2.1. Suppose that $p>1$. If $u_{0}^{\prime} \geq 0$ in $[0,1]$, then the blow-up occurs only at $x=1$.

PROOF. Suppose that there is a blow-up point $a \in[0,1)$. Then there is a sequence $\left\{\left(x_{n}, t_{n}\right)\right\}$ such that $x_{n} \rightarrow a, t_{n} \rightarrow T$, and $u\left(x_{n}, t_{n}\right) \rightarrow \infty$ as $n \rightarrow \infty$. Fix a constant $d \in(a, 1)$. By comparing the solution $u$ with the function

$$
u\left(x_{n}, t_{n}\right) \sin [\pi(x-d) /(1-d)] \exp \left\{-[\pi /(1-d)]^{2}\left(t-t_{n}\right)\right\}
$$

for each $n$ sufficiently large, it is easy to show that

$$
u(x, t) \geq u\left(x_{n}, t_{n}\right) \sin [\pi(x-d) /(1-d)] \exp \left\{-[\pi /(1-d)]^{2}\left(t-t_{n}\right)\right\}
$$

for $x \in[d, 1]$ and $t \geq t_{n}$. Hence

$$
\lim _{t \rightarrow T} u(x, t)=\infty
$$

uniformly over $x \in[b, c]$ for any compact subset $[b, c]$ of $(d, 1)$. 
Now, we fix a compact subset $[b, c]$ of $(d, 1)$. We take any $r \in(1, p)$ and consider the function

$$
J(x, t)=u_{x}(x, t)-g(x) u^{r}(x, t),
$$

where $g(x)=\varepsilon \sin [\pi(x-b) /(c-b)]$ for some $\varepsilon>0$ to be determined. Using (2.4), there is a $t_{0} \in(0, T)$ such that

$$
J_{t}-J_{x x}-\left(p u^{p-1}+2 r g^{\prime} u^{r-1}\right) J \geq 0
$$

in $[b, c] \times\left[t_{0}, T\right)$ for any $\varepsilon>0$. By choosing $\varepsilon>0$ sufficiently small such that $J\left(x, t_{0}\right) \geq 0$ for any $x \in[b, c]$, it follows from the maximum principle that $J \geq 0$ in $[b, c] \times\left[t_{0}, T\right)$. Hence we have

$$
u^{-r}(x, t) u_{x}(x, t) \geq g(x) \text { in }[b, c] \times\left[t_{0}, T\right) .
$$

An integration of (2.6) leads to a contradiction. Hence the theorem follows.

If $p \leq 1$, then $q>1$, since $\max \{p, q\}>1$.

LEMMA 2.2. Let $0<p \leq 1$. If $u_{0}^{\prime} \geq 0$ in $[0,1]$, then $x=1$ is the only blow-up point.

Proof. Since $u_{0}^{\prime}(1)>0$, there is a constant $\delta \in(0,1)$ such that $u_{0}^{\prime}>0$ in $[1-\delta, 1]$. Set

$$
\eta=\inf _{1-\delta \leq x \leq 1}\left\{u_{0}^{\prime}(x) / u_{0}^{q}(x)\right\}, \quad M=(q-p) \sup _{[1-\delta, 1] \times[0, T)} u^{p-1} .
$$

Then $0<\eta \leq 1$ and $0<M<\infty$. Choose a positive integer $n \geq 3$ such that $n \geq M$ and a positive number $\varepsilon<\min \left\{\eta, \delta^{n}\right\}$. Define $g(x)=\left(x-1+\varepsilon^{1 / n}\right)^{n}$ if $1-\varepsilon^{1 / n} \leq x \leq 1$; $g(x)=0$, otherwise. It is easy to see that $g \in C^{2}([0,1])$ and satisfies

$$
0 \leq g \leq \varepsilon, \quad g^{\prime} \geq 0, \quad g^{\prime \prime} \geq 0, \quad g^{\prime \prime} \geq M g .
$$

Then, by using the fact $q>1$ and the maximum principle, it is easy to show that

$$
g(x) u^{q}(x, t) \leq u_{x}(x, t)
$$

for $0 \leq x \leq 1$ and $0 \leq t<T$. Hence

$$
u^{-q}(x, t) u_{x}(x, t) \geq g(x)
$$

for $0 \leq x \leq 1$ and $0 \leq t<T$. An integration of (2.8) shows that $u$ cannot blow up at any point $x<1$. This proves the lemma.

Indeed, the condition $u_{0}^{\prime} \geq 0$ in $[0,1]$ can be removed if $0<p \leq 1$.

THEOREM 2.3. If $0<p \leq 1$, then $x=1$ is the only blow-up point.

Proof. We first extend the function $u(x, t)$ to $w(x, t)$ defined on $[-1,1] \times[0, T)$ so that $w(x, t)=u(x, t)$ and $w(-x, t)=w(x, t)$ for $x \in[0,1]$ and $t \in[0, T)$. Then $w$ satisfies

$$
\begin{aligned}
& w_{t}=w_{x x}+w^{p}, \quad x \in(-1,1), \quad 0<t<T, \\
& w_{x}(-1, t)=-w^{q}(-1, t), \quad w_{x}(1, t)=w^{q}(1, t), \quad 0<t<T .
\end{aligned}
$$


Arguing as Lemma 1.2 of [9], there exists $t^{*} \in(0, T)$ such that

$$
n(t):=\#\left\{a \in[-1,1] \mid w_{x}(a, t)=0\right\}
$$

is a constant for all $t \geq t^{*}$. Moreover, there are $C^{1}$ functions $s_{0}(t), \ldots, s_{ \pm l}(t), l \geq 0$, from $\left[t^{*}, T\right)$ to $[-1,1]$ such that

$$
\begin{aligned}
& s_{-l}(t)<\cdots<s_{0}(t)<\cdots<s_{l}(t), \quad s_{0}(t) \equiv 0, \\
& \left\{a \in[-1,1] \mid w_{x}(a, t)=0\right\}=\left\{s_{-l}(t), \ldots, s_{0}(t), \ldots, s_{l}(t)\right\} \quad \text { for } t \geq t^{*},
\end{aligned}
$$

and the limit $s_{i}:=\lim _{t \uparrow T} s_{i}(t)$ exists for all $i$. Since $n(t)$ is constant in $\left[t^{*}, T\right)$, it follows from Theorem $\mathrm{C}$ of [1] that $w_{x x}\left(s_{i}(t), t\right) \neq 0$ for all $t \in\left[t^{*}, T\right)$. Note that for each $i$ there is a fixed sign for $w_{x x}\left(s_{i}(t), t\right)$ for all $t \in\left[t^{*}, T\right)$. Also, it suffices to consider the so-called maximum curve, i.e., the curve for which $w_{x x}\left(s_{i}(t), t\right)<0$ on $\left[t^{*}, T\right)$.

If $l=0$, then $u_{x}(x, t)>0$ on $(0,1] \times\left[t^{*}, T\right)$. Hence the conclusion follows from Lemma 2.2. Suppose that $l>0$. Set $m_{i}(t):=w\left(s_{i}(t), t\right)$. Notice that $m_{i}^{\prime}(t)<m_{i}(t)^{p}$ on $\left[t^{*}, T\right)$, if $w_{x x}\left(s_{i}(t), t\right)<0$ on $\left[t^{*}, T\right)$. Since $0<p \leq 1, m_{i}(t)$ remains bounded near $T$. This implies that $w$ cannot blow up at any point in $(-1,1)$. The theorem is proved.

3. Some a priori estimates. In this section, we will derive some a priori estimates which will be used in Section 5 to prove the time asymptotic results. Let $u$ be the solution of (2.1) - (2.3) with blow-up time $T$. From now on we shall always assume that $u_{0}^{\prime} \geq 0$ in $[0,1]$, so that by the maximum principle we have $u_{x}>0$ in $(0,1] \times(0, T)$. Notice that $u(1, t)=\max _{0 \leq x \leq 1} u(x, t)$.

The following lemma is given in [11] under the assumption $u_{0}^{\prime \prime}+u_{0}^{p} \geq a>0$ in $[0,1]$. Indeed, we have the following lemma.

LEMMA 3.1. If $u_{0}^{\prime \prime}+u_{0}^{p} \geq 0$ in $[0,1]$, then $u_{t} \geq 0$ in $[0,1] \times[0, T)$.

Proof. Set $v=u_{t}$. Then $v$ satisfies

$$
\begin{aligned}
& v_{t}=v_{x x}+p u^{p-1} v, \quad 0<x<1, \quad 0<t<T, \\
& v_{x}(0, t)=0, \quad v_{x}(1, t)=q u^{q-1}(1, t) v(1, t), \quad 0<t<T, \\
& v(x, 0)=u_{0}^{\prime \prime}+u_{0}^{p} \geq 0, \quad 0 \leq x \leq 1 .
\end{aligned}
$$

For any fixed $\tau \in(0, T)$, let

$$
L=\max _{0 \leq x \leq 1,0 \leq t \leq \tau}\left\{\frac{1}{2} q u^{q-1}(x, t)\right\}, \quad M=2 L+4 L^{2}+\max _{0 \leq x \leq 1,0 \leq t \leq \tau}\left\{p u^{p-1}(x, t)\right\} .
$$

Set $w(x, t)=e^{-M t-L x^{2}} v(x, t)$. Then $w$ satisfies

$$
\begin{aligned}
& w_{t}=w_{x x}+4 L x w_{x}+c w, \quad 0<x<1, \quad 0<t \leq \tau, \\
& w_{x}(0, t)=0, \quad w_{x}(1, t)=d w(1, t), \quad 0<t \leq \tau, \\
& w(x, 0) \geq 0, \quad 0 \leq x \leq 1,
\end{aligned}
$$

where $c=c(x, t) \leq 0$ and $d=d(t) \leq 0$. By the maximum principle, we obtain that $w \geq 0$ in $[0,1] \times[0, \tau]$. Hence the lemma follows. 
Recall from [11] that if $u_{t} \geq 0$, then there are positive constants $c$ and $A$ such that

$$
c(T-t)^{-\alpha} \leq u(1, t) \leq A(T-t)^{-\alpha},
$$

where the exponent is given by

$$
\alpha=\left\{\begin{array}{cl}
1 /(p-1) & \text { if } p \geq 2 q-1 \\
1 /[2(q-1)] & \text { if } p<2 q-1
\end{array}\right.
$$

Hereafter we shall assume that $u_{0}^{\prime} \geq 0$ and $u_{0}^{\prime \prime}+u_{0}^{p} \geq 0$ in $[0,1]$. Therefore, we have $u_{x} \geq 0$ and $u_{t} \geq 0$. We now make the following Giga-Kohn transformation

$$
\begin{aligned}
& y=\frac{1-x}{\sqrt{T-t}}, \quad s=-\ln (T-t), \\
& w(y, s)=(T-t)^{\alpha} u(x, t),
\end{aligned}
$$

where $\alpha$ is defined as in (3.1). Let

$$
W=\left\{(y, s) \mid 0<y<e^{s / 2}, s>-\ln T\right\} .
$$

Then for $p>2 q-1$ we have

$$
\begin{aligned}
& w_{s}=w_{y y}-\frac{y}{2} w_{y}-\alpha w+w^{p} \quad \text { in } W, \\
& w_{y}(0, s)=-e^{\gamma s} w(0, s)^{q}, \quad w_{y}\left(e^{s / 2}, s\right)=0, \quad s>-\ln T, \\
& w(y,-\ln T)=T^{\alpha} u_{0}(1-y \sqrt{T}), \quad 0 \leq y \leq 1 / \sqrt{T},
\end{aligned}
$$

where $\gamma=[(2 q-1)-p] /[2(p-1)]<0$; for $p=2 q-1$ we have

$$
\begin{aligned}
& w_{s}=w_{y y}-\frac{y}{2} w_{y}-\alpha w+w^{p} \quad \text { in } W, \\
& w_{y}(0, s)=-w(0, s)^{q}, \quad w_{y}\left(e^{s / 2}, s\right)=0, \quad s>-\ln T, \\
& w(y,-\ln T)=T^{\alpha} u_{0}(1-y \sqrt{T}), \quad 0 \leq y \leq 1 / \sqrt{T},
\end{aligned}
$$

while for $p<2 q-1$ we have

$$
\begin{aligned}
& w_{s}=w_{y y}-\frac{y}{2} w_{y}-\alpha w+e^{\sigma s} w^{p} \quad \text { in } W, \\
& w_{y}(0, s)=-w(0, s)^{q}, \quad w_{y}\left(e^{s / 2}, s\right)=0, \quad s>-\ln T, \\
& w(y,-\ln T)=T^{\alpha} u_{0}(1-y \sqrt{T}), \quad 0 \leq y \leq 1 / \sqrt{T},
\end{aligned}
$$

where $\sigma=[p-(2 q-1)] /[2(q-1)]<0$.

We have the following a priori estimates for $w$.

LEMMA 3.2. $w$ and $w_{y}$ are bounded in $\bar{W}$.

PROOF. The fact that $w$ is bounded follows from (3.1).

It follows from Lemma 3.1 that $u_{x x} \geq-u^{p}$ in $[0,1] \times[0, T)$. Multiplying the above inequality by $u_{x} \geq 0$ and integrating it from $x$ to 1 , we obtain

$$
u_{x}^{2}(x, t) \leq u^{2 q}(1, t)+\frac{2}{p+1} u^{p+1}(1, t) .
$$


Note that $w_{y}(y, s)=-(T-t)^{\alpha+1 / 2} u_{x}(x, t)$.

Recall (3.1). For $p \geq 2 q-1$, it follows from (3.13) and Lemma 3.1 that

$$
\begin{aligned}
w_{y}^{2}(y, s) & \leq(T-t)^{2 \alpha+1} u^{2 q}(1, t)+\frac{2}{p+1}(T-t)^{2 \alpha+1} u^{p+1}(1, t) \\
& =\left[(T-t)^{\alpha} u(1, t)\right]^{p+1} u^{2 q-1-p}(1, t)+\frac{2}{p+1}\left[(T-t)^{\alpha} u(1, t)\right]^{p+1} \\
& \leq A^{p+1} u_{0}^{2 q-1-p}(1)+A^{p+1} .
\end{aligned}
$$

For $p<2 q-1$, it also follows from (3.13) and Lemma 3.1 that

$$
\begin{aligned}
w_{y}^{2}(y, s) & \leq(T-t)^{2 \alpha+1} u^{2 q}(1, t)+\frac{2}{p+1}(T-t)^{2 \alpha+1} u^{p+1}(1, t) \\
& =\left[(T-t)^{\alpha} u(1, t)\right]^{2 q}+\frac{2}{p+1}\left[(T-t)^{\alpha} u(1, t)\right]^{2 q} u^{p-2 q+1}(1, t) \\
& \leq A^{2 q}+\frac{2}{p+1} A^{2 q} u_{0}^{p-2 q+1}(1) .
\end{aligned}
$$

Hence the lemma is proved.

Lemma 3.3. There is a positive constant $C$ such that $\left|w_{s}(y, s)\right| \leq C(1+y)$ and $\left|w_{y y}(y, s)\right| \leq C(1+y)$ in $\bar{W}$.

Proof. It follows from Lemma 3.2 that $\left|w_{s}(y, s)-w_{y y}(y, s)\right| \leq C(1+y)$ in $\bar{W}$ for some positive constant $C$. The lemma follows by applying the standard theory of parabolic equations, e.g., Theorem 6.44, Theorem 4.30 and Theorem 4.31 in [10].

4. Self-similar solution. In this section, we shall study the self-similar solution of (1.1) $-(1.2)$ for the case $p=2 q-1$, i.e., $q=(p+1) / 2$. We are concerned with the existence and uniqueness of global positive monotone decreasing solution of the initial value problem (P):

$$
\begin{aligned}
& w^{\prime \prime}-\frac{1}{2} y w^{\prime}-\alpha w+w^{p}=0, \quad y>0, \\
& w^{\prime}(0)=-w^{q}(0),
\end{aligned}
$$

where $w=w(y)$ and $\alpha=1 /(p-1)$. We always assume that $p>1$. The existence result has been obtained before by Wang and Wang in [12]. Here we present a different proof for the existence. Some of the lemmas will be useful for the proof of uniqueness.

Given any $\eta>0$, there is a unique local solution $w(y ; \eta)$ of $(4.1)-(4.2)$ with $w(0)=\eta$. Let $\rho(y)=\exp \left\{-y^{2} / 4\right\}$ and $f(w)=-\alpha w+w^{p}$. Then $w$ satisfies

$$
\left(\rho w^{\prime}\right)(y)=-\eta^{q}-\int_{0}^{y} \rho(s) f(w(s)) d s .
$$


Let $\kappa$ be the unique positive solution of $f(w)=0$. Note that $w^{\prime}<0$ as long as $w \geq \kappa$. Set

$$
\kappa_{0}=\left(\frac{p+1}{p+3}\right)^{\alpha} \kappa .
$$

Note that $0<\kappa_{0}<\kappa$.

LEMMA 4.1. Let $\eta \geq \kappa_{0}$. Then $w^{\prime}<0$ as long as $w>0$.

PROOF. Let

$$
G(y)=\frac{1}{2}\left[w^{\prime}(y)\right]^{2}+F(w(y)),
$$

where $F(w)=\int_{\kappa}^{w} f(s) d s$. Note that

$$
G(0) \geq F(0) \text { if and only if } \eta \geq \kappa_{0} .
$$

Since

$$
G^{\prime}(y)=\frac{1}{2} y\left[w^{\prime}(y)\right]^{2},
$$

and the problem $(\mathrm{P})$ has no non-trivial constant solution, $G$ is strictly increasing.

If $w$ is not monotone decreasing, then there is the first critical point $y_{0}>0$ of $w$ such that $w^{\prime}\left(y_{0}\right)=0$ and $w>0$ in $\left[0, y_{0}\right]$. Notice that $w\left(y_{0}\right)<\kappa$. Hence

$$
G\left(y_{0}\right)=F\left(w\left(y_{0}\right)\right)<F(0) .
$$

On the other hand, by (4.5) we have $G(0) \geq F(0)$, since $\eta \geq \kappa_{0}$. This implies that $G(0)>$ $G\left(y_{0}\right)$, a contradiction. Therefore, the lemma follows.

Suppose that $w>0$ and $w^{\prime}<0$ in $[0, \infty)$. Let $l=\lim _{y \rightarrow \infty} w(y)$. Then $l \in[0, \kappa)$ and there is a sequence $\left\{y_{n}\right\}$ such that $w^{\prime}\left(y_{n}\right) \rightarrow 0$ as $n \rightarrow \infty$. Dividing Equation (4.1) by $y$ and integrating it from 1 to $y_{n}$ for any $n$ large, as $n \rightarrow \infty$, this leads to a contradiction, if $l \in(0, \kappa)$. Hence $l=0$.

LEMMA 4.2. For $\eta \geq \kappa_{0}$, the solution $w$ is monotone decreasing to zero at some finite $R$.

Proof. Otherwise, by Lemma 4.1 and the above observation, $w(y) \rightarrow 0$ as $y \rightarrow \infty$ and there is a sequence $\left\{y_{n}\right\}$ such that $w^{\prime}\left(y_{n}\right) \rightarrow 0$ as $n \rightarrow \infty$. Then $G\left(y_{n}\right) \rightarrow F(0)$ as $n \rightarrow$ $\infty$. Since $G$ is monotone increasing, its limit must be greater than $G(0)$, i.e., $F(0)>G(0)$, a contradiction to (4.5). This proves the lemma.

We now turn to the case when $\eta$ is small. First, let $\eta_{0}$ be a positive constant such that $-f(w) \geq \alpha w / 2$ for all $w \in\left[0, \eta_{0}\right]$. Notice that $\eta_{0}<\kappa$. Choose $\eta_{1} \in\left(0, \eta_{0}\right)$ such that $\eta^{1-q}>e^{1 / 4}$ for all $\eta \in\left(0, \eta_{1}\right)$. Now, given any fixed $\eta \in\left(0, \eta_{1}\right)$, suppose that $w^{\prime}<0$ in $[0, R]$ and $w(R)=0$ for some $R=R(\eta)>0$. Since, by (4.3), $\rho(y) w^{\prime}(y) \geq-\eta^{q}$ for all $y \in[0, R)$, we have

$$
\eta=-\int_{0}^{R} w^{\prime}(s) d s \leq \eta^{q} R e^{R^{2} / 4}
$$


Let $g(y)=y e^{y^{2} / 4}$. Since $g$ is strictly monotone increasing, we conclude that $R=R(\eta)>1$, if $\eta<\eta_{1}$.

LEMMA 4.3. There is a small positive constant $\eta_{*}$ such that $w^{\prime}$ vanishes before $w$ vanishes, if $\eta<\eta_{*}$.

PROOF. Let $\eta_{*}$ be a positive constant such that $\eta_{*}<\eta_{1}$ and

$$
\eta^{1-q}>\frac{1+\alpha / 4}{\alpha / 2} e^{1 / 4} \text { for all } \eta \in\left(0, \eta_{*}\right) .
$$

Suppose that there is an $\eta \in\left(0, \eta_{*}\right)$ such that the lemma does not hold. Then the corresponding solution $w$ must have the property that $w^{\prime}<0$ in $\left[0, y_{0}\right]$ for some $y_{0}>1$. From (4.3) it follows that $\rho(y) w^{\prime}(y) \geq-\eta^{q}$ for all $y \in\left[0, y_{0}\right]$ and so

$$
w(y)=\eta+\int_{0}^{y} w^{\prime}(s) d s \geq \eta-\eta^{q} y e^{y^{2} / 4} \text { for all } y \in\left[0, y_{0}\right] .
$$

Then from (4.3), (4.7), and noting that $\eta<\eta_{0}$, we obtain that

$$
\begin{aligned}
\rho(y) w^{\prime}(y) & \geq-\eta^{q}+\frac{\alpha}{2} \int_{0}^{y} \rho(s) w(s) d s \\
& \geq-\eta^{q}+\frac{\alpha}{2} \int_{0}^{y} e^{-s^{2} / 4}\left[\eta-\eta^{q} s e^{s^{2} / 4}\right] d s \\
& \geq-\left(1+\frac{\alpha}{4} y^{2}\right) \eta^{q}+\frac{\alpha}{2} y e^{-y^{2} / 4} \eta
\end{aligned}
$$

for all $y \in\left(0, y_{0}\right)$. In particular, for $y=1$ we have

$$
e^{-1 / 4} w^{\prime}(1) \geq-\left(1+\frac{\alpha}{4}\right) \eta^{q}+\frac{\alpha}{2} e^{-1 / 4} \eta>0,
$$

since $\eta<\eta_{*}$. This is a contradiction and the lemma is proved.

Now, we define

$$
\begin{aligned}
& I_{1}=\{\eta>0 \mid w(y ; \eta) \text { is decreasing to zero at some finite } R\} \\
& I_{2}=\left\{\eta>0 \mid w^{\prime}(y ; \eta) \text { vanishes before } w(y ; \eta) \text { vanishes }\right\}
\end{aligned}
$$

Notice that $w$ and $w^{\prime}$ cannot vanish at the same time. Hence $I_{1}$ and $I_{2}$ are disjoint. Lemmas 4.2 and 4.3 imply that $\left[\kappa_{0}, \infty\right) \subset I_{1}$ and $\left(0, \eta_{*}\right) \subset I_{2}$.

LEMMA 4.4. The set $I_{2}$ is open.

Proof. Let $\eta_{0} \in I_{2}$. Then $\eta_{0}<\kappa_{0}<\kappa$ and there is the first point $y_{0}>0$ such that $w_{0}>0$ in $\left[0, y_{0}\right], w_{0}^{\prime}<0$ in $\left[0, y_{0}\right)$ and $w_{0}^{\prime}\left(y_{0}\right)=0$, where $w_{0}(y)=w\left(y ; \eta_{0}\right)$. Since $w_{0}^{\prime \prime}\left(y_{0}\right)>0$, there is a positive constant $\delta$ such that $w_{0}^{\prime}(y)>0$ for $y \in\left(y_{0}, y_{0}+\delta\right]$. Let $\varepsilon>0, \varepsilon<w_{0}\left(y_{0}\right) / 2$, and $\varepsilon<w_{0}^{\prime}\left(y_{0}+\delta\right) / 2$. By the continuous dependence of initial value, there is a positive constant $\gamma$ such that $\left|w(y ; \eta)-w_{0}(y)\right|<\varepsilon$ and $\left|w^{\prime}(y ; \eta)-w_{0}^{\prime}(y)\right|<\varepsilon$ for all $y \in\left[0, y_{0}+\delta\right]$, if $\eta \in\left(\eta_{0}-\gamma, \eta_{0}+\gamma\right)$. This implies that $\left(\eta_{0}-\gamma, \eta_{0}+\gamma\right) \subset I_{2}$ and so $I_{2}$ is open. 
To prove that $I_{1}$ is open, we consider the quantity

$$
H(y)=\alpha w(y)+\frac{1}{2} y w^{\prime}(y) .
$$

Then $H$ satisfies the equation

$$
H^{\prime}(y)=\frac{1}{2} y H(y)+\left(\frac{1}{2}+\alpha\right) w^{\prime}(y)-\frac{1}{2} y w^{p}(y) .
$$

Suppose that $H\left(y_{0}\right)<0$ for some $y_{0} \geq 0$. Then $w^{\prime}\left(y_{0}\right)<0$ by (4.8) and $H^{\prime}\left(y_{0}\right)<0$ by (4.9). Hence, by (4.9) again, $H^{\prime}(y)<0$ and $H(y)<0$ for all $y \geq y_{0}$ as long as $w>0$.

LEMMA 4.5. The set $I_{1}$ is open.

PROOF. First, we claim that if there is a point $y_{0} \geq 0$ such that $H\left(y_{0}\right)<0$, then $w$ is decreasing after $y_{0}$ and vanishes at some finite $R>y_{0}$. Otherwise, if $w>0$ in $[0, \infty)$, then $H(y)<0$ and $H^{\prime}(y)<0$ for all $y \geq y_{0}$. Hence there is a positive constant $\delta$ such that $H(y) \leq-\delta$ for all $y \geq y_{0}$. By an integration, we obtain that

$$
w(y) \leq\left(y_{0} / y\right)^{2 \alpha} w\left(y_{0}\right)-\frac{\delta}{\alpha}+\frac{\delta}{\alpha}\left(y_{0} / y\right)^{2 \alpha} \rightarrow-\frac{\delta}{\alpha} \quad \text { as } \quad y \rightarrow \infty,
$$

a contradiction.

Now, let $\eta_{0} \in I_{1}$ and $w_{0}(y)=w\left(y ; \eta_{0}\right)$. Then there is a finite $R_{0}>0$ such that $w_{0}^{\prime}<0$ and $w_{0}>0$ in $\left[0, R_{0}\right)$. Since $w_{0}\left(R_{0}\right)=0$ and $w_{0}^{\prime}\left(R_{0}\right)<0$, there is a positive constant $\delta$ such that $H_{0}\left(R_{0}-\delta\right)<0$, where $H_{0}(y)=\alpha w_{0}(y)+y w_{0}^{\prime}(y) / 2$. It follows from the theory of continuous dependence on initial value that there is a positive constant $\gamma$ such that $w(y ; \eta)>$ $0, w^{\prime}(y ; \eta)<0$ for $y \in\left[0, R_{0}-\delta\right]$, and $H\left(R_{0}-\delta\right)<0$, if $\eta \in\left(\eta_{0}-\gamma, \eta_{0}+\gamma\right)$. Then $w$ is decreasing after $R_{0}-\delta$ and vanishes at some finite $R>R_{0}-\delta$, if $\eta \in\left(\eta_{0}-\gamma, \eta_{0}+\gamma\right)$. Hence the lemma is proved.

We now state and prove an existence theorem as follows.

THEOREM 4.6. There is a global positive monotone decreasing solution of $(\mathrm{P})$.

Proof. Set $\bar{\eta}=\inf I_{1}$. Then the corresponding solution $\bar{w}(y)=w(y ; \bar{\eta})$ must be a global positive monotone decreasing solution of $(\mathrm{P})$.

Indeed, for any $\eta \notin I_{1} \cup I_{2}$, the corresponding solution $w(y ; \eta)$ is a global positive monotone decreasing solution of (P) satisfying $w(y ; \eta) \rightarrow 0$ as $y \rightarrow \infty$.

We have from Lemma 4.2 the estimate $\bar{\eta}<\kappa_{0}$. Also, the initial value $\eta<\kappa_{0}$ for any global positive monotone decreasing solution of $(\mathrm{P})$. To derive a better estimate, we need the following generalized version of Pohozaev Identity, which is inspired by Lemma 2.1 of [13] (see also [14]).

LEMMA 4.7. Suppose $w(y)$ is a solution of $(\mathrm{P})$ and define

$$
J(y):=\rho(y)\left(w^{\prime}(y)\right)^{2}-\frac{y}{2} \rho(y) w^{\prime}(y) w(y)+\left(\frac{1}{4}-\alpha\right) \rho(y) w^{2}(y)+\frac{2}{p+1} \rho(y) w^{p+1}(y),
$$


where $\rho(y)=\exp \left[-y^{2} / 4\right]$. Then the following identity holds:

$J(y)=\frac{p+3}{p+1} w^{p+1}(0)+\frac{p-5}{4(p-1)} w^{2}(0)+\int_{0}^{y} s \rho(s)\left\{\frac{p-1}{2(p+1)} w^{p-1}(s)-\frac{1}{8}\right\} w^{2}(s) d s$.

Proof. Differentiating $J(y)$ and using (4.1), we obtain

$$
J^{\prime}(y)=y \rho(y)\left\{\frac{p-1}{2(p+1)} w^{p-1}(y)-\frac{1}{8}\right\} w^{2}(y) .
$$

Integrating $J^{\prime}(y)$ from 0 to $y$ and noting that

$$
J(0)=\frac{p+3}{p+1} w^{p+1}(0)+\frac{p-5}{4(p-1)} w^{2}(0),
$$

we get the desired identity.

COROLlaRY 4.8. Suppose that $w(y)$ is a global positive solution of $(\mathrm{P})$ satisfying $w(y) \rightarrow 0$ as $y \rightarrow \infty$. Then

$$
\int_{0}^{\infty} s \rho(s)\left\{\frac{1}{8}-\frac{p-1}{2(p+1)} w^{p-1}(s)\right\} w^{2}(s) d s=\frac{p+3}{p+1} w^{p+1}(0)+\frac{p-5}{4(p-1)} w^{2}(0) .
$$

PROOF. Since $w(y)$ is a global positive solution of $(\mathrm{P})$ and $\lim _{y \rightarrow \infty} w(y)=0$, there is a sequence $y_{n} \rightarrow \infty$ such that $\lim _{n \rightarrow \infty} w^{\prime}\left(y_{n}\right)=0$. Using Lemma 4.7, (4.10) follows.

Define $f_{1}(w)=\left\{1 / 8-[(p-1) /(2(p+1))] w^{p-1}\right\} w^{2}$ and let $\bar{\kappa}=(\alpha / 2)^{\alpha}$. Then it is easy to check that

$$
\max _{w \in[0, \infty)} f_{1}(w)=f_{1}(\bar{\kappa}) .
$$

The following lemma gives an upper bound for any global positive solution of $(\mathrm{P})$ which tends to zero as $y \rightarrow \infty$.

LEMMA 4.9. Suppose that $w(y)$ is a global positive solution of $(\mathrm{P})$ with $w(0)=\eta$ such that $w(y) \rightarrow 0$ as $y \rightarrow \infty$. Then $\eta<\bar{\kappa}$. In particular, we have $\bar{\eta}<\bar{\kappa}$.

PROOF. For contradiction, we assume that $\eta \geq \bar{\kappa}$. It follows from the definition of $\bar{\kappa}$ that

On the other hand, since $w(0)=\eta \geq \bar{\kappa}$, we have

$$
\int_{0}^{\infty} s \rho(s) f_{1}(w(s)) d s<\int_{0}^{\infty} s \rho(s) f_{1}(\bar{\kappa}) d s=\frac{p-1}{4(p+1)} \bar{\kappa}^{2} .
$$

$$
\begin{aligned}
\frac{p+3}{p+1} w^{p+1}(0)+\frac{p-5}{4(p-1)} w^{2}(0) & \geq \frac{p+3}{2(p+1)(p-1)} w^{2}(0)+\frac{p-5}{4(p-1)} w^{2}(0) \\
& =\frac{p-1}{4(p+1)} w^{2}(0) \geq \frac{p-1}{4(p+1)} \bar{\kappa}^{2},
\end{aligned}
$$

a contradiction to (4.10). This completes the proof.

THEOREM 4.10. If $1<p \leq 2$, then there is a unique global positive monotone decreasing solution of $(\mathrm{P})$. 
PROOF. For contradiction, we suppose that there are two distinct global positive monotone decreasing solutions $w_{1}$ and $w_{2}$ of (P). Note that $w_{i}(y) \rightarrow 0$ as $y \rightarrow \infty$ for $i=1,2$.

First, we claim that $w_{1}$ and $w_{2}$ must intersect each other at least once. Multiplying the equation

$$
\left(\rho w_{i}^{\prime}\right)^{\prime}(y)=-\rho(y) f\left(w_{i}(y)\right), \quad i=1,2,
$$

by $w_{2}$ for $i=1$; by $w_{1}$ for $i=2$, respectively, and integrating by parts, we end up with

$$
\int_{0}^{\infty} \rho(s) w_{1}(s) w_{2}(s)\left(w_{1}^{p-1}(s)-w_{2}^{p-1}(s)\right) d s=w_{1}(0) w_{2}(0)\left(w_{2}^{q-1}(0)-w_{1}^{q-1}(0)\right),
$$

using $w_{i}^{\prime}(0)=-w_{i}^{q}(0), i=1,2$. Hence they must intersect each other at least once.

Without loss of generality, we may assume that $w_{1}(y)>w_{2}(y)$ in $\left[0, y_{0}\right)$ and $w_{1}\left(y_{0}\right)=$ $w_{2}\left(y_{0}\right)$ for some $y_{0}>0$. Then we have $w_{1}^{\prime}\left(y_{0}\right)<w_{2}^{\prime}\left(y_{0}\right)$. Hence there exists $y_{1}>y_{0}$ such that $w_{1}\left(y_{1}\right)<w_{2}\left(y_{1}\right)$. Define $v(y):=w_{1}(y)-w_{2}(y)$. Then it follows from (4.1) that $v(y)$ satisfies

$$
v^{\prime \prime}-\frac{y}{2} v^{\prime}+\left[p \xi^{p-1}(y)-\alpha\right] v=0,
$$

for some $\xi(y) \in\left[\min \left\{w_{1}(y), w_{2}(y)\right\}, \max \left\{w_{1}(y), w_{2}(y)\right\}\right]$. Since $\lim _{y \rightarrow \infty} w_{i}(y)=0, i=$ 1,2 , there exists $y_{2}>y_{1}$ such that $\left|v\left(y_{2}\right)\right|<\left|v\left(y_{1}\right)\right| / 2$.

Now, let $\bar{y} \in\left[0, y_{2}\right]$ be a minimal point of $v$ in $\left[0, y_{2}\right]$. Then $\bar{y} \in\left(0, y_{2}\right)$ and $v(\bar{y})<0$. Since $\bar{y}$ is an interior extreme point of $v$, we have

$$
v^{\prime}(\bar{y})=0, \quad v^{\prime \prime}(\bar{y}) \geq 0 .
$$

From Lemma 4.9 and $\xi(\bar{y}) \leq \max \left\{w_{1}(0), w_{2}(0)\right\}$, it follows that

$$
p \xi^{p-1}(\bar{y})-\alpha<0,
$$

if $1<p \leq 2$. Then, by (4.11), (4.12) and (4.13), we obtain that

$$
\begin{aligned}
0= & v^{\prime \prime}(\bar{y})-\frac{\bar{y}}{2} v^{\prime}(\bar{y})+\left[p \xi^{p-1}(\bar{y})-\alpha\right] v(\bar{y}) \\
& \geq\left[p \xi^{p-1}(\bar{y})-\alpha\right] v(\bar{y}) \\
& >0
\end{aligned}
$$

a contradiction. This completes the proof.

We conjecture that Theorem 4.10 should hold for any $p>1$. Unfortunately we are unable to prove it now, so we left it as an open problem.

5. Time asymptotic analysis. In this section, we shall study the time asymptotic of the solutions of the problem $(2.1)-(2.3)$ for various cases. The method is the same as the one used in [8] with some modifications. Hence we shall only give the outline of the proofs.

THEOREM 5.1. For $p>2 q-1$, we have

$$
(T-t)^{\alpha} u(1-y \sqrt{T-t}, t) \rightarrow \kappa
$$

as $t \rightarrow T$ uniformly for $y \in[0, C]$ for any $C>0$. Here $\alpha=1 /(p-1)$ and $\kappa=\alpha^{\alpha}$. 
Proof. As in [8], we take any increasing sequence $\left\{s_{j}\right\}$ in $(0, \infty)$ such that $s_{j+1}-s_{j} \rightarrow$ $\infty$ as $j \rightarrow \infty$. For each $j \in N$, we define

$w_{j}(y, s)=w\left(y, s+s_{j}\right) \quad$ for all $(y, s) \in W_{j} \equiv\left\{(y, s) \mid 0 \leq y \leq e^{\left(s+s_{j}\right) / 2}, s \geq-s_{j}-\ln T\right\}$.

Note that $\bigcup_{j=1}^{\infty} W_{j}=[0, \infty) \times \boldsymbol{R}$ and $W_{1} \subset W_{2} \subset \cdots$. Recall Lemmas 3.2 and 3.3. By the Ascoli-Arzela Theorem and a diagonal process, we can get a subsequence (still denoted by $\left.w_{j}\right)$ such that $w_{j}(y, s) \rightarrow w_{\infty}(y, s)$ as $j \rightarrow \infty$ uniformly on any compact subset of $[0, \infty) \times \boldsymbol{R}$ and that for any integer $m$ we have $w_{j, y}(y, m) \rightarrow w_{\infty, y}(y, m)$ as $j \rightarrow \infty$ pointwise for $y \in[0, \infty)$ for some function $w_{\infty}$ defined on $[0, \infty) \times \boldsymbol{R}$. It is easy to see that $w_{\infty}$ is a classical solution of the equation

$$
w_{s}=w_{y y}-\frac{1}{2} y w_{y}-\alpha w+w^{p} \quad \text { in }[0, \infty) \times \boldsymbol{R} .
$$

Now, we claim that $w_{\infty, s}(y, s) \equiv 0$ in $[0, \infty) \times \boldsymbol{R}$. Introduce the energy function

$$
E[w](s)=\frac{1}{2} \int_{0}^{s} \rho w_{y}^{2} d y+\frac{\alpha}{2} \int_{0}^{s} \rho w^{2} d y-\frac{1}{p+1} \int_{0}^{s} \rho w^{p+1} d y,
$$

where $\rho(y)=e^{-y^{2} / 4}$. By a simple computation, we get

$$
-\frac{d}{d s} E[w](s)=\int_{0}^{s} \rho w_{s}^{2} d y-G(s),
$$

where

$$
\begin{aligned}
G(s)= & \rho(s)\left\{\frac{1}{2} w_{y}^{2}(s, s)+\frac{\alpha}{2} w^{2}(s, s)-\frac{1}{p+1} w^{p+1}(s, s)+w_{y}(s, s) w_{s}(s, s)\right\} \\
& +\exp \left\{\frac{(2 q-1)-p}{2(p-1)} s\right\} w(0, s)^{q} w_{s}(0, s) .
\end{aligned}
$$

Let $s_{0}=\max \{2 \ln 2,-\ln T\}$. Note that

$$
\left\{(y, s) \mid 0 \leq y \leq s, s \geq s_{0}\right\} \subseteq \bar{W} .
$$

Integrating both sides of (5.1) from $m+s_{j}$ to $m+s_{j+1}$ for any $m, j \in \boldsymbol{Z}$ with $m+s_{j} \geq s_{0}$, we obtain

$$
\begin{aligned}
\int_{m+s_{j}}^{m+s_{j+1}} & \int_{0}^{s} \rho(y) w_{s}^{2}(y, s) d y d s \\
& =E_{m+s_{j}}[w]\left(m+s_{j}\right)-E_{m+s_{j+1}}[w]\left(m+s_{j+1}\right)+\int_{m+s_{j}}^{m+s_{j+1}} G(s) d s .
\end{aligned}
$$

By a change of variable, we get

$$
\begin{aligned}
\int_{m}^{m+s_{j+1}-s_{j}} & \int_{0}^{s+s_{j}} \rho(y) w_{j, s}^{2}(y, s) d y d s \\
& =E_{m+s_{j}}\left[w_{j}\right](m)-E_{m+s_{j+1}}\left[w_{j+1}\right](m)+\int_{m+s_{j}}^{m+s_{j+1}} G(s) d s .
\end{aligned}
$$


Since

it follows that

$$
|G(s)| \leq C \exp \left\{\frac{(2 q-1)-p}{2(p-1)} s\right\}(1+s),
$$

$$
\int_{s_{0}}^{\infty}|G(s)| d s<\infty \text {. }
$$

Proceeding as in [8], we get

$$
\int_{m}^{M} \int_{0}^{\infty} \rho w_{\infty, s}^{2} d y d s=0 \text { for all } m, M \in Z, \quad m<M .
$$

Hence $w_{\infty, s} \equiv 0$ and so $w_{\infty}(y, s)=w_{\infty}(y)$ for all $y \in[0, \infty)$ and $s$.

Note that $w_{\infty}(0)>0$. Also, from $w_{j, y}(0, s)=-e^{\gamma\left(s+s_{j}\right)} w_{j}(0, s)^{q}$, where

$$
\gamma=[(2 q-1)-p] /[2(p-1)]<0,
$$

it follows that $w_{\infty}^{\prime}(0)=0$. Therefore, $w_{\infty}$ is a bounded positive global solution of

$$
w^{\prime \prime}-\frac{1}{2} y w^{\prime}-\alpha w+w^{p}=0
$$

and so $w_{\infty} \equiv \kappa$ (cf. [8]). Since the sequence $\left\{s_{j}\right\}$ is arbitrary, the theorem follows.

Recall from [6] that there is a unique bounded positive global solution (denoted by $V(y)$ ) of

$$
w^{\prime \prime}-\frac{1}{2} y w^{\prime}-\alpha w=0, \quad w^{\prime}(0)=-w^{q}(0) .
$$

THEOREM 5.2. For $p<2 q-1$, we have

$$
(T-t)^{\alpha} u(1-y \sqrt{T-t}, t) \rightarrow V(y)
$$

as $t \rightarrow T$ uniformly for $y \in[0, C]$ for any $C>0$. Here $\alpha=1 /[2(q-1)]$.

Proof. Let $s_{j}, w_{j}, w_{\infty}$ be defined as in Theorem 5.1. Then it is easy to see that $w_{\infty}$ is a classical solution of

$$
w_{s}=w_{y y}-\frac{1}{2} y w_{y}-\alpha w, \quad y \in[0, \infty), \quad s \in \boldsymbol{R} .
$$

Next, we introduce the energy function

$$
E[w](s)=\frac{1}{2} \int_{0}^{s} \rho w_{y}^{2} d y+\frac{\alpha}{2} \int_{0}^{s} \rho w^{2} d y-\frac{1}{q+1} w^{q+1}(0, s) .
$$

Proceeding as in the proof of Theorem 5.1, we obtain that $w_{\infty, s} \equiv 0$ and so $w_{\infty}(y, s)=$ $w_{\infty}(y)$. Since $w_{y}(0, s)=-w^{q}(0, s)$, we get $w_{\infty}^{\prime}(0)=-w_{\infty}^{q}(0)$. Recall $w_{\infty}(0)>0$. Hence $w_{\infty}(y)=V(y)$ and the theorem follows.

Finally, we shall consider the critical case, i.e., the case $p=2 q-1$. Suppose that $\bar{w}(y)$ (as defined in Section 4) is the unique global positive monotone decreasing solution of (4.1). Then the same argument as above leads to the following conclusion. Note that $w_{y}<0$ for $y \geq 0$. Hence the limit function satisfies $w_{\infty}^{\prime} \leq 0$. 
THEOREM 5.3. Let $p=2 q-1$. If $1<p \leq 2$, then we have

$$
(T-t)^{\alpha} u(1-y \sqrt{T-t}, t) \rightarrow \bar{w}(y)
$$

as $t \rightarrow T$ uniformly for $y \in[0, C]$ for any $C>0$. Here $\alpha=1 /(p-1)$.

6. Complete blow-up. Suppose that the solution $u$ of the problem (1.1)-(1.3) blows up at the finite time $T$ and $x=1$ is the only blow-up point. This is the case if $\max \{p, q\}>1$ and $u_{0}^{\prime} \geq 0$ in $[0,1]$. Let

$$
f^{(n)}(s)=\min \left\{s^{q}, n^{q}\right\}, \quad g^{(n)}(s)=\min \left\{s^{p}, n^{p}\right\}, \quad s \geq 0, \quad n \in \boldsymbol{N},
$$

and let $u^{(n)}$ be the solution of the problem $\left(B^{(n)}\right)$ :

$$
\begin{aligned}
& u_{t}^{(n)}=u_{x x}^{(n)}+g^{(n)}\left(u^{(n)}\right), \quad x \in(0,1), \quad t>0, \\
& u_{x}^{(n)}(0, t)=0, u_{x}^{(n)}(1, t)=f^{(n)}\left(u^{(n)}(1, t)\right), \quad t>0, \\
& u^{(n)}(x, 0)=u_{0}(x), \quad x \in[0,1] .
\end{aligned}
$$

We shall follow the method used in [5] to prove that the blow-up is complete, i.e., as $n \rightarrow \infty$, $u^{(n)}(x, t) \rightarrow \infty$ for all $(x, t) \in[0,1] \times(T, \infty)$.

Let $K=\max _{0 \leq x \leq 1} u_{0}(x)$. Since $f^{(n)}$ and $g^{(n)}$ are locally Lipschitz in $(0, K]$ and $u_{0}^{\prime}(1)=f^{(n)}\left(u_{0}(1)\right)$ for $n>K$, the solution $u^{(n)}$ of $\left(B^{(n)}\right)$ is $C^{1}$ up to the boundary.

Suppose that $v^{(n)}$ is a positive smooth supersolution and $w^{(n)}$ is a smooth subsolution of $\left(B^{(n)}\right)$. Then it is easy to show by the maximum principle that $v^{(n)} \geq w^{(n)}$ for $0 \leq x \leq 1$, $t>0$, if $n>K$. Note that the function $K+\left(n^{p}+n^{q}\right) t+n^{q} x^{2} / 2$ is a supersolution of $\left(B^{(n)}\right)$. Therefore, for any positive integer $n>K$, the problem $\left(B^{(n)}\right)$ has a unique positive global (in time) solution $u^{(n)}$ such that $u^{(n)} \leq u^{(n+1)}$ for $(x, t) \in[0,1] \times[0, \infty)$ and $u^{(n)} \leq u$ for $(x, t) \in[0,1] \times[0, T)$.

Now, we define

$$
v(x, t)=\lim _{n \rightarrow \infty} u^{(n)}(x, t), \quad 0 \leq x \leq 1, \quad t>0 .
$$

Then we can show that $v(x, t)=u(x, t)$ for $0 \leq t<T$. Note that $v(1, T)=\infty$. Furthermore, we have

LEMMA 6.1. If $q \geq 1$, then $v(1, t)=\infty$ for all $t \geq T$.

PROOF. For any $M>1$, there is a smooth function $U$ such that $U(1)=M, U^{\prime}(1)=$ $M^{q}, U(\xi)=0$, and $U^{\prime \prime}+U^{p}=0$ in $(\xi, 1]$ for some $\xi \in(0,1)$, since $q \geq 1$. We extend the function $U$ to be linear on $[0, \xi]$ so that $U \in C^{2}([0,1])$. Let $M>\max \left\{1, u_{0}(1),\left\|u_{0}^{\prime}\right\|_{\infty}^{1 / q}\right\}$. Then $u_{0}$ intersects $U$ exactly once.

Since $v(1, T)=\infty$, there is a positive integer $k>K$ such that $u^{(k)}\left(1, t_{0}\right)>M$ for some $t_{0} \in(0, T)$. Then there is $t_{1} \in\left(0, t_{0}\right)$ such that $u^{(k)}\left(1, t_{1}\right)=M$ and $u^{(k)}(1, t)<M$ for all $t \in\left[0, t_{1}\right)$. Since $u^{(k)}(0, t)>U(0)$ and $u^{(k)}(1, t)<U(1)$ for all $t \in\left[0, t_{1}\right)$, it implies that $u^{(k)}(\cdot, t)$ intersects $U$ at least once. Note that

$$
\left(u^{(k)}-U\right)_{t}=\left(u^{(k)}-U\right)_{x x}+c(x, t)\left(u^{(k)}-U\right), \quad c(x, t)=\frac{g^{(k)}\left(u^{(k)}\right)+U_{x x}}{u^{(k)}-U} .
$$


Since $u^{(k)}(x, t)$ is bounded away from zero in $[0,1] \times\left[0, t_{1}\right)$, the function $c(x, t)$ is bounded. Applying Theorem D of [1], $u^{(k)}(\cdot, t)$ intersects $U$ exactly once for $t<t_{1}$. Let $s^{(k)}(t)$ be the function such that

$$
\left(u^{(k)}-U\right)\left(s^{(k)}(t), t\right)=0 \text { for all } t \in\left[0, t_{1}\right) .
$$

Applying Theorem D of [1] again, we have

$$
\left(u^{(k)}-U\right)_{x}\left(s^{(k)}(t), t\right) \neq 0 \text { for all } t \in\left[0, t_{1}\right)
$$

Therefore, by the Implicit Function Theorem, the function $s^{(k)}(t)$ is continuous in $\left[0, t_{1}\right)$.

Now, we will show that

$$
u^{(k)}\left(x, t_{1}\right) \geq U(x) \text { for all } x \in[0,1] .
$$

To prove (6.4), we consider two cases. First, we suppose that $\lim _{t \rightarrow t_{1}^{-}} s^{(k)}(t)$ exists. we claim that $\lim _{t \rightarrow t_{1}^{-}} s^{(k)}(t)=1$. For contradiction, we assume that $0 \leq \lim _{t \rightarrow t_{1}^{-}} s^{(k)}(t)<1$. Recall that $\left(u^{(k)}-U\right)\left(1, t_{1}\right)=0$ and $\left(u^{(k)}-U\right)(x, t) \leq 0$ for all $x \in\left[s^{(k)}(t), 1\right]$ and $t \in\left[0, t_{1}\right]$. By the Hopf Boundary Point Lemma, $\left(u^{(k)}-U\right)_{x}\left(1, t_{1}\right)>0$, a contradiction. Since

$$
\left(u^{(k)}-U\right)(x, t) \geq 0, \quad x \in\left[0, s^{(k)}(t)\right], \quad t \in\left[0, t_{1}\right),
$$

by letting $t \rightarrow t_{1}$, the inequality (6.4) follows.

Next, we suppose that $\lim _{t \rightarrow t_{1}^{-}} s^{(k)}(t)$ does not exist. We assume that

$$
a \equiv \liminf _{t \rightarrow t_{1}^{-}} s^{(k)}(t)<\limsup _{t \rightarrow t_{1}^{-}} s^{(k)}(t) \equiv b .
$$

Then $\xi<a<b \leq 1$. It is easy to see that $\left(u^{(k)}-U\right)\left(x, t_{1}\right)=0$ for all $x \in[a, b]$ and $\left(u^{(k)}-U\right)\left(x, t_{1}\right)>0$ for all $x \in[0, a)$. If $b=1$, then (6.4) follows immediately. Suppose that $b<1$. For contradiction, we assume that $\left(u^{(k)}-U\right)\left(x_{0}, t_{1}\right)<0$ for some $x_{0} \in(b, 1)$. Since $\lim \sup _{t \rightarrow t_{1}^{-}} s^{(k)}(t)=b<x_{0}$, there exists $t_{2} \in\left[0, t_{1}\right)$ such that $s^{(k)}(t)<x_{0}$ for all $t \in\left(t_{2}, t_{1}\right)$. Hence

$$
\left(u^{(k)}-U\right)(x, t) \leq 0, \quad \text { for all }(x, t) \in\left\{\left[x_{0}, 1\right] \times\left[t_{2}, t_{1}\right]\right\} \cup\left\{\left[s^{(k)}(t), 1\right] \times\left[0, t_{2}\right]\right\} .
$$

It follows from the Hopf Boundary Point Lemma that $\left(u^{(k)}-U\right)_{x}\left(1, t_{1}\right)>0$, a contradiction. Hence (6.4) follows.

Since $u^{(k)}(\xi, t)>U(\xi)$ for all $t \geq t_{1}$, it follows from the maximum principle that $u^{(k)}(x, t) \geq U(x)$ for all $(x, t) \in[\xi, 1] \times\left[t_{1}, \infty\right)$. In particular, $u^{(n)}(1, t) \geq M$ for all $t \geq T$ and $n \geq k$. Hence $v(1, t)=\infty$ for all $t \geq T$ and the lemma follows. 
Now, by the representation formula of solution of $\left(B^{(n)}\right)$,

$$
\begin{aligned}
u^{(n)}(x, t)= & \int_{0}^{1} u^{(n)}\left(y, t_{1}\right) \Gamma\left(x, t ; y, t_{1}\right) d y+\int_{t_{1}}^{t} f^{(n)}\left(u^{(n)}(1, \tau)\right) \Gamma(x, t ; 1, \tau) d \tau \\
& -\int_{t_{1}}^{t} u^{(n)}(1, \tau) \Gamma_{y}(x, t ; 1, \tau) d \tau+\int_{t_{1}}^{t} u^{(n)}(0, \tau) \Gamma_{y}(x, t ; 0, \tau) d \tau \\
& +\int_{t_{1}}^{t} \int_{0}^{1} g^{(n)}\left(u^{(n)}(y, \tau)\right) \Gamma(x, t ; y, \tau) d y d \tau
\end{aligned}
$$

for $x \in(0,1)$ and $t>t_{1} \geq 0$, where

$$
\Gamma(x, t ; y, \tau)=\frac{1}{\sqrt{4 \pi(t-\tau)}} \exp \left\{-\frac{(x-y)^{2}}{4(t-\tau)}\right\},
$$

and the jump relation of $u^{(n)}(0, t)$

$$
\begin{aligned}
\frac{1}{2} u^{(n)}(0, t)= & \int_{0}^{1} u^{(n)}\left(y, t_{1}\right) \Gamma\left(0, t ; y, t_{1}\right) d y+\int_{t_{1}}^{t} f^{(n)}\left(u^{(n)}(1, \tau)\right) \Gamma(0, t ; 1, \tau) d \tau \\
& -\int_{t_{1}}^{t} u^{(n)}(1, \tau) \Gamma_{y}(0, t ; 1, \tau) d \tau+\int_{t_{1}}^{t} \int_{0}^{1} g^{(n)}\left(u^{(n)}(y, \tau)\right) \Gamma(0, t ; y, \tau) d y d \tau
\end{aligned}
$$

for $t>t_{1} \geq 0$, we conclude that $v(x, t) \equiv \infty$ for all $t>T$. This proves that the blow-up is complete when $q \geq 1$.

\section{REFERENCES}

[ 1 ] S. Angenent, The zeroset of a solution of a parabolic equation, J. Reine Angew. Math. 390 (1988), 79-96.

[2 2 M. ChLebík AND M. Fila, Some recent results on blow-up on the boundary for the heat equation, B. Bojarski et al. (ed.), Proceedings of the Minisemester, Warsaw, Poland, (Warsaw, 1998), 61-71, Banach Center Publ. 52, Polish Acad. Sci., Warsaw, 2000.

[3] A. De Pablo, F. Quirós And J. D. Rossi, Asymptotic simplification for a reaction-diffusion problem with a nonlinear boundary condition, IMA J. Appl. Math. 67 (2002), 69-98.

[ 4 ] M. Fila AND J. Filo, Blow-up on the boundary: A survey S. Janeczko et al. (ed.), Singularities and differential equations, (Warsaw, 1996), 67-78, Banach Center Publ. 33, Polish Acad. Sci., Warsaw, 1996.

[ 5 ] M. FILA AND J.-S. Guo, Complete blow-up and incomplete quenching for the heat equation with a nonlinear boundary condition, Nonlinear Anal. 48 (2002), 995-1002.

[6] M. FILA AND P. QuitTNer, The blow-up rate for the heat equation with a nonlinear boundary condition, Math. Methods Appl. Sci. 14 (1991), 197-205.

[ 7 ] A. FRIEDMAN AND B. MCLEOD, Blow-up of positive solutions of semilinear heat equations, Indiana Univ. Math. J. 34 (1985), 425-447.

[ 8 ] Y. Giga AND R. V. KoHN, Asymptotically self-similar blow-up of semilinear heat equations, Comm. Pure Appl. Math. 38 (1985), 297-319.

[ 9 ] J.-S. Guo, On the quenching behavior of the solution of a semilinear parabolic equation, J. Math. Anal. Appl. 151 (1990), 58-79.

[10] G. M. Lieberman, Second Order Parabolic Differential Equations, World Scientific, Singapore, 1996.

[11] Z. LIN AND M. WANG, The blow-up properties of solutions to semilinear heat equations with nonlinear boundary conditions, Z. Angew. Math. Phys. 50 (1999), 361-374. 
[12] M. WANG AND X. WANG, Existence of positive solutions to a nonlinear initial problem, Nonlinear Anal. 44 (2001), 1133-1136.

[13] E. Yanagida, Uniqueness of positive radial solutions of $\Delta u+g(r) u+h(r) u^{p}=0$ in $R^{n}$, Arch. Rational Mech. Anal. 115 (1991), 257-274.

[14] E. YANAGIDA, Structure of positive radial solutions of Matukuma's equation, Japan J. Indust. Appl. Math. 8 (1991), 165-173.

SHENG-CHEN FU:

Department of MATHEMATICAL SCIEnCES

NATIONAL ChENGCHi UNIVERSiTy

64 SEC. 2, ZHI-NAN ROAD

TAIPEI 116

TAIWAN
JONG-SHENQ GUO AND JE-CHIANG TSAI:

DEPARTMENT OF MATHEMATICS

NATIONAL TAIWAN NORMAL UNIVERSITY

88, SEC. 4, Ting CHOU RoAD

TAIPEI 117

TAIWAN 